\title{
GLOBAL BIFURCATION THEOREMS FOR NONLINEARLY PERTURBED OPERATOR EQUATIONS
}

\author{
BY JOHN MACBAIN
}

Communicated by R. K. Miller, February 3, 1976

1. Introduction. The author [2], [3], and [4] has previously studied the equation

$$
L u=\lambda u+H(\lambda, u)
$$

in a real Banach space $B$ where $L$ is linear and $H$ is compact and $o(\|u\|)$ is uniformly on bounded $\lambda$ intervals. In that setting, if $\lambda_{0}$ is an isolated normal eigenvalue of $L$ having odd algebraic multiplicity, then $\left(\lambda_{0}, 0\right) \in R \times B$ is a bifurcation point for (1). Moreover, a continuous branch of solutions emanates from each of these points and obeys a threefold alternative.

This paper combines methods of the author and Stuart [7] to show that similar results hold if $H(\lambda, u)$ is merely continuous and $o(\|u\|)$ uniformly on bounded $\lambda$ intervals.

2. Preliminaries. In this paper all work is a real Banach space $B . L$ denotes a linear operator densely defined in $B$, and $H$ represents a continuous operator that is $o(\|u\|)$ near $u=0$ uniformly on bounded $\lambda$ intervals. Define the essential spectrum of $L$ as the members of the spectrum of $L$ which are not isolated normal eigenvalues of $L$ and denote this set by $e(L)$.

We consider a normal eigenvalue $\lambda_{0}$ of $L$. Let

$$
\alpha_{\lambda_{0}}=\sup \left\{\gamma \mid \gamma \in e(L), \gamma<\lambda_{0}\right\} \quad \text { and } \quad \beta_{\lambda_{0}}=\inf \left\{\gamma \mid \gamma \in e(L), \gamma>\lambda_{0}\right\}
$$

respectively if the corresponding sup or inf exists. Otherwise, set $\alpha_{\lambda_{0}}=-\infty$ and/ or $\beta_{\lambda_{0}}=+\infty$. Assume for now that $\alpha_{\lambda_{0}}$ and $\beta_{\lambda_{0}}$ are both finite. For $\epsilon>0$, the only members of the spectrum of $L$ in $\left(\alpha_{\lambda_{0}}+\epsilon, \beta_{\lambda_{0}}-\epsilon\right)$ are normal eigenvalues of $L$. If $P_{\epsilon}$ denotes the projector onto the direct sum of the eigenspaces of these eigenvalues and $Q_{\epsilon}=I-P_{\epsilon}$, then it has been shown [2], [3] and [4] that

$$
u=\frac{\left(L-\mu_{0}\right) P_{\epsilon} u}{\lambda-\mu_{0}}+\left((L-\lambda)^{-1} Q_{\epsilon}-\frac{P_{\epsilon}}{\lambda-\mu_{0}}\right) H(\lambda, u)
$$

is equivalent to (1) for $\lambda$ in $\left[\alpha_{\lambda_{0}}+\epsilon, \beta_{\lambda_{0}}-\epsilon\right]$ and $\mu_{0}$ any member of the resolvent of $L$ not lying in $\left(\alpha_{\lambda_{0}}, \beta_{\lambda_{0}}\right)\left((L-\lambda)^{-1}\right.$ is defined on $\left.Q_{\epsilon} B\right)$.

AMS (MOS) subject classifications (1970). Primary 46N05.

Copyright $\odot$ 1976, American Mathematical Society 
Define

$$
M(\lambda, \gamma)=\sup \left\{\frac{\|H(\lambda, u)\|}{\|u\|} \mid 0<\|u\| \leqslant \gamma\right\}
$$

Clearly $\lim _{\gamma \rightarrow 0} M(\lambda, \gamma)=0$.

3. Results. Consider equation (1) in a real Banach space $B$, with $L$ linear and $H$ continuous, $o(\|u\|)$ uniformly on bounded intervals.

THEOREM I. Let $\lambda_{0}$ be an isolated eigenvalue of $L$ having odd algebraic multiplicity. Then $\left(\lambda_{0}, 0\right)$ is a bifurcation point for (2) and emanating from that point there is a maximal continuous branch of solutions $C_{\lambda_{0}}$ that must obey the following alternative:

(a) $C_{\lambda_{0}}$ is unbounded, or

(b) $C_{\lambda_{0}}$ is bounded and for each $\delta>0, C_{\lambda_{0}}$ meets the surface

$$
\begin{aligned}
& S_{\epsilon}=\left\{(\lambda, u)\|\|(L-\lambda)^{-1} Q_{\epsilon} \| M(\lambda, \gamma)=1,\right. \\
& \left.\|u\|=\gamma, \quad \alpha_{\lambda_{0}}+\epsilon<\lambda<\beta_{\lambda_{0}}-\epsilon\right\} \quad \text { for some } \epsilon, 0<\epsilon<\delta,
\end{aligned}
$$

(c) $C_{\lambda_{0}}$ is bounded, $\overline{C_{\lambda_{0}}}$ does not meet $S_{\epsilon}$ for all $\epsilon \in(0, \delta)$ for some $\delta>0$, and $C_{\lambda_{0}} \cap\{0 \times B\}=\left\{\lambda_{0}, \lambda_{1}, \ldots, \lambda_{n}\right\}$, each a distinçt normal eigenvalue of $L$, and the sum of their algebraic multiplicities being even.

Proof. Assume for now that $\alpha_{\lambda_{0}}$ and $\beta_{\lambda_{0}}$ are finite, and that $C_{\lambda_{0}}$ does not obey any of the three alternatives. Then $C_{\lambda_{0}}$ consists of pairs $(\lambda, u)$ with $\alpha_{\lambda_{0}}+2 \epsilon<\lambda<\beta_{\lambda_{0}}-2 \epsilon$ for some $\epsilon>0$. Using this $\epsilon$, one sees that the operators on the right side of (2) are a $k$-set contraction with

$$
k=\left\|(L-\lambda)^{-1} Q_{\epsilon}\right\| M(\lambda, \gamma) \text { for each } \lambda \in\left(\alpha_{\lambda_{0}}+\epsilon, \beta_{\lambda_{0}}-\epsilon\right),
$$

and $u$ with $\|u\| \leqslant \gamma$. We may further assume that $C_{\lambda_{0}}$ does not meet $S_{\epsilon}$. If $S_{\epsilon}$ is viewed in $\mathbf{R} \times\|B\|$, we see that $k<1$ below $S_{\epsilon}$. Techniques developed in [2] and [6] which employ degree theory for $k$-set contractions [5] lead to a contradiction.

In the case that $\alpha_{\lambda_{0}}$ or $\beta_{\lambda_{0}}$ are infinite, the result is obtained using an iterative procedure.

In the case that $L$ is selfadjoint, the results are simpler. Let $d(\lambda)=$ $\min \left(\lambda-\alpha_{\lambda_{0}}, \beta_{\lambda_{0}}-\lambda\right)$.

COROLlary 1. If the assumptions of Theorem I hold and if, moreover, $L$ is selfadjoint, then alternatives (b) and (c) are equivalent to

(b') $C_{\lambda_{0}}$ is bounded and $\overline{C_{\lambda_{0}}}$ meets

$$
S=\left\{(\lambda, u) \mid \frac{M(\lambda, \gamma)}{d(\lambda)}=1,\|u\|=\gamma, \alpha_{\lambda_{0}}<\lambda<\beta_{\lambda_{0}}\right\}
$$


(c') $C_{\lambda_{0}}$ is bounded, $\overline{C_{\lambda_{0}}}$ does not meet $S$, and $C_{\lambda_{0}} \cap\{0 \times B\}=\left\{\lambda_{0}\right.$, $\left.\lambda_{1}, \ldots, \lambda_{n}\right\}$, each a distinct normal eigenvalue of $L$, and the sum of their algebraic multiplicities being even.

PROOF. With $L$ being selfadjoint and $\alpha_{\lambda_{0}}+\epsilon<\lambda<\beta_{\lambda_{0}}-\epsilon$,

$$
\left\|(L-\lambda)^{-1} Q_{\epsilon}\right\|=\left\|(L-\lambda)^{-1}\left|Q_{\epsilon} B\|\| Q_{\epsilon}\|=\|(L-\lambda)^{-1}\right| Q_{\epsilon} B\right\| .
$$

As $\epsilon$ goes to $0,\left\|(L-\lambda)^{-1} \mid Q_{\epsilon} B\right\|$ approaches $\left\|(L-\lambda)^{-1} Q_{0} B\right\|=1 / d(\lambda)$ and $S_{\epsilon}$ approaches $S$.

\section{REFERENCES}

1. M. A. Krasnosel'skii, Topological methods in the theory of nonlinear integral equations, GITTL, Moscow, 1956; English transl., Macmillan, New York, 1964. MR 20 \# 3464; 28 \# 2414.

2. J. A. MacBain, Local and global bifurcation from normal eigenvalues, $\mathrm{Ph} . \mathrm{D}$. Thesis, Purdue Univ., 1974.

3. - Global bifurcation theorems for noncompact operators, Bull. Amer. Math. Soc. 80 (1974), 1005-1009. MR 50 \# 14403. 63 (1976).

4. - Local and global bifurcation from normal eigenvalues, Pacific J. Math.

5. R. D. Nussbaum, The fixed point index and fixed point theorems for $k$-set contractions, Ph.D. Thesis, Chicago, Ill., 1969.

6. P. H. Rabinowit $\mathrm{z}$, Some aspects of nonlinear eigenvalue problems, Rocky Mountain Consortium Sympos. on Nonlinear Eigenvalue Problems (Santa Fe, N. M., 1971), Rocky Mountain J. Math. 3 (1973), 161-202. MR 47 \# 9383.

7. C. A. Stuart, Some bifurcation theory for $k$-set contractions, Proc. London Math. Soc. (3) 27 (1973), $531-550$.

DEPARTMENT OF MATHEMATICS, AIR FORCE INSTITUTE OF TECHNOLOGY, WRIGHT-PATTERSON AIR FORCE BASE, DAYTON, OHIO 45433 